\title{
CONCENTRACIÓN DE CADMIO EN AGUA, SEDIMENTOS, Eichhornia crassipes Y Pomacea canaliculata EN EL RÍO GUAYAS (ECUADOR) Y SUS AFLUENTES
}

\author{
Concentration of cadmium in water, sediments, Eichhornia crassipes and Pomacea canaliculata in the Guayas
}

(Ecuador) river and tributaries

\author{
Mariuxi MERO ${ }^{1}$, Beatriz PERNÍA ${ }^{2 *}$, Nelson RAMÍREZ-PRADO ${ }^{2}$, Kenya BRAVO $^{2}$, Lissette RAMÍREZ ${ }^{2}$, \\ Erwin LARRETA ${ }^{3}$ y Fidel EGAS ${ }^{4}$
}

${ }^{1}$ Instituto de Investigaciones de Recursos Naturales, Facultad de Ciencias Naturales, Universidad de Guayaquil, Av. Raúl Gómez Lince s/n y Av. Juan Tanca Marengo, Guayaquil, Ecuador, C.P. 090150

${ }^{2}$ Facultad de Ciencias Naturales, Universidad de Guayaquil, Av. Raúl Gómez Lince s/n y Av. Juan Tanca Marengo, Guayaquil, Ecuador, C.P. 090150

${ }^{3}$ Escuela Superior Politécnica del Litoral, ESPOL, Facultad de Ingeniería en Ciencias de la Tierra, Campus Gustavo Galindo, Km 30.5 Vía Perimetral, Guayaquil, Ecuador, C.P. 09015863

${ }^{4}$ Gobierno Provincial del Guayas, calle Pichincha y General Juan Illingworht 107, Guayaquil, Ecuador, C.P. 090313

*Autor para correspondencia: beatrizpernia@gmail.com

(Recibido marzo 2018; aceptado octubre 2018)

Palabras clave: contaminación, biomonitores, metal pesado, río Daule, río Babahoyo

\section{RESUMEN}

En Ecuador existen evidencias de contaminación por cadmio en el Golfo de Guayaquil, sin embargo, poco se conoce sobre la contaminación por este metal en sus ríos afluentes. El propósito de la investigación fue evaluar la contaminación por cadmio en agua, sedimentos, jacinto de agua Eichhornia crassipes y en el caracol Pomacea canaliculata en los ríos Guayas, Daule y Babahoyo. Para ello, se muestrearon nueve estaciones en total, se tomaron cinco muestras de agua, cinco de sedimentos y cinco de organismos por localidad, se determinaron las concentraciones de $\mathrm{Cd}$ y los factores de bioconcentración ( $\mathrm{FBC}$ ). Además, se midieron los parámetros $\mathrm{pH}$, temperatura y salinidad. El Cd en agua no fue detectable en los sitios estudiados, mientras que en sedimentos ocho de las nueve estaciones excedieron los límites máximos permisibles según la norma ecuatoriana y la norma canadiense de calidad ambiental. Sin embargo, las concentraciones biodisponibles del metal en el sedimento fueron inferiores al nivel de efecto probable (PEL) y al valor de referencia canadiense, minimizando los posibles daños a la biota. El jacinto de agua acumuló mayores concentraciones de Cd que el gasterópodo y presentó $\mathrm{FBC}$ mayores a uno, indicando que es un buen biomonitor de $\mathrm{Cd}$, a diferencia del caracol. También se evidenció correlación entre los parámetros físicos y químicos y la disponibilidad del metal en el sedimento y su acumulación en los organismos. Se concluye que existe contaminación por $\mathrm{Cd}$ en los tres ríos y se propone el monitoreo anual de estos, utilizando la especie E. crassipes como biomonitora.

Key words: contamination, biomonitor, heavy metal, Daule river, Babahoyo river 


\begin{abstract}
In Ecuador there is evidence of contamination by $\mathrm{Cd}$ in the Gulf of Guayaquil, however, little is known about the contamination by this metal in its tributaries. Evaluating the contamination by cadmium in water, sediments, flora as Eichhornia crassipes and fauna as Pomacea canaliculata in the Guayas river and the tributaries, Daule and Babahoyo rivers was the main purpose of this research. Nine stations were sampled, five samples of water, sediment and organisms were taken on each location. Cd concentrations and bioconcentration factors (BCF) were determined. In addition, $\mathrm{pH}$, temperature and salinity parameters were measured. The concentrations of $\mathrm{Cd}$ in water were not detectable, while in sediments eight of a total of nine stations exceeded the maximum permissible limits according to the Ecuadorian standard and the Canadian Environmental Quality Guidelines. However, the bioavailable concentrations of the metal in the sediment were lower than the probable effect level (PEL) and the Canadian reference value, minimizing possible damage to the biota. The water hyacinth accumulated higher concentrations of $\mathrm{Cd}$ than the gastropod and presented $\mathrm{BCF}$ greater than one, indicating that it is a good biomonitor of Cd, unlike the snail. Correlation was also observed between the physical and chemical parameters and the availability of the metal in the sediment and its accumulation in the organisms. It is concluded that there is contamination by $\mathrm{Cd}$ in the three rivers and that the E. crassipes can be used as biomonitor of bioavailability of the metal in the ecosystem.
\end{abstract}

\section{INTRODUCCIÓN}

El cadmio $(\mathrm{Cd})$ es un metal tóxico liberado al ambiente por fuentes naturales y antrópicas (Castrillo et al. 2012). Dentro de las fuentes naturales se encuentran la actividad volcánica, los incendios forestales y el transporte por el viento de partículas del suelo (Filipič 2012). Las fuentes antrópicas son la minería, el uso de fertilizantes fosfatados, la fundición de metales, la quema de combustibles fósiles, la fabricación de baterías, pigmentos, cemento, y plásticos (Pernía et al. 2008).

El Cd liberado al ambiente tiende a acumularse en los suelos haciéndose disponible para las plantas, entrando así a la cadena alimenticia (Castrillo et al. 2012). Las plantas representan el primer eslabón en la cadena trófica y algunas especies son capaces de acumular grandes cantidades de $\mathrm{Cd}$, lo que expone a los animales herbívoros a almacenar cantidades apreciables de este metal (Burger 2008). Resulta imprescindible estudiar el contenido de cadmio en plantas y animales herbívoros en ecosistemas potencialmente contaminados con este metal.

De estos ecosistemas, los ríos han sido los más afectados ya que reciben una gran cantidad de aguas servidas domésticas e industriales. El Cd llega a los ríos donde se deposita en los sedimentos y es absorbido por las plantas y los animales. Una alta concentración de metales en los ríos podría traer como consecuencia la reducción en el crecimiento y supervivencia de la población de peces, delfines de río y del zooplancton, así como generar la extinción de algunas especies (Paul 2017). Se ha descrito que el Cd afecta los riñones, pulmones, esqueleto, testículos y sistema nervioso central de los animales y humanos, generando hipocalcemia, diabetes, osteoporosis y cáncer (Burger 2008, Clemens et al. 2013). Por otro lado, en las plantas, este metal produce inhibición de crecimiento, clorosis, estrés oxidativo, peroxidación lipídica, inhibición de enzimas, oxidación de proteínas y daño al ADN (Castrillo et al. 2012, Pernía et al. 2008).

A nivel mundial se ha descrito contaminación por Cd en sedimentos del río Casqueiro en Brasil (Harris y Santos 2000), el río Yamuna en la India (Kaushik et al. 2009) y en estuarios tales como la Ciénaga Grande de Santa Marta en Colombia (Perdomo et al. 1999) el Golfo de Cariaco en Venezuela (Fuentes et al. 2010), Sudarban en Bangladesh (Islam et al. 2017) y estanques de Wyścigien Polonia (Wojtkowska et al. 2015), entre otros lugares.

En Ecuador existen evidencias de contaminación por Cd en el Golfo de Guayaquil, el cual representa una de las áreas más productivas en la costa noroeste de América del Sur (Fernández-Cadena et al. 2014). En este sentido, Proaño (2016) encontró altos valores de este metal en sedimentos de la Reserva Ecológica Manglares Churute (5.95-15.97 mg/kg). De igual forma, Mero et al. (2012) determinaron en Puerto el Morro concentraciones de 2.99-4.99 mg $/ \mathrm{kg}$ y Fernández-Cadena et al. (2014) en el estero Salado $0.50-12.00 \mathrm{mg} / \mathrm{kg}$. Sin embargo, existe poca 
información sobre los niveles de este metal en los ríos afluentes que llegan al Golfo de Guayaquil.

El Golfo de Guayaquil se ha industrializado, incrementando el número de empresas de plástico, metalmecánicas y camaroneras que arrojan sus aguas residuales a los ríos sin tratamiento previo. Además de ello, a su alrededor existen numerosas hectáreas de cultivo de arroz y caña de azúcar, donde se utilizan fertilizantes que podrían contener $\mathrm{Cd}$ (Muñoz 2017).

El río Guayas es el principal afluente del Golfo de Guayaquil y se forma por la confluencia de los ríos Daule y Babahoyo. En los tres ríos abundan las especies Eichhornia crassipes y Pomacea canaliculata. La planta acuática E. crassipes es abundante en la mayoría de los ríos de la zona, está ampliamente distribuida y ha sido reportada como una especie acumuladora de $\mathrm{Cd}$ por lo que representa un excelente biomonitor. Por otro lado, el gasterópodo $P$. canaliculata es un herbívoro abundante, de poca movilidad, consume a la especie E. crassipes y fue descrito como biomonitor de $\mathrm{Cd}$ en los ríos en estudio (Ramírez et al. 2016). Es por ello, que el propósito de la investigación fue evaluar los niveles de Cd en agua, sedimentos, jacinto de agua (Eichhornia crassipes) y el caracol (Pomacea canaliculata) en el río Guayas y sus afluentes.

\section{MATERIALES Y MÉTODOS}

La cuenca del río Guayas, es una de las 72 cuencas del Pacífico y está formada por la confluencia de varios ríos entre los que destacan el Daule y el Babahoyo, los cuales atraviesan extensas zonas de varias provincias y confluyen en el río Guayas en la ciudad de Guayaquil (Huayamabe 2013).

Para la investigación se establecieron nueve estaciones de muestreo, tres en el río Guayas, tres en el río Daule y tres en el río Babahoyo. Las estaciones se seleccionaron bajo los criterios de predicción de zonas con abundante vegetación, con base en imágenes satelitales y en su facilidad de acceso para la colecta de muestras (Fig. 1). Las coordenadas geográficas de cada estación de muestreo fueron determinadas utilizando un GPS modelo Triton (Magellan ${ }^{\circledR}$ ).

\section{Muestreo}

Para el cumplimiento de los objetivos se realizaron salidas de campo para la colecta de aguas, sedimentos, organismos animales y vegetales considerados biomonitores en los ríos Guayas, Daule y Babahoyo en los sitios ya establecidos (Fig. 1).
En cada río se muestrearon tres estaciones y en cada una de las estaciones se realizó un transecto donde se colectaron cinco muestras de agua, sedimento, gasterópodos y plantas a una distancia de $10 \mathrm{~m}$ entre ellas; con un total de 15 muestras por sustrato y un total de 60 muestras por río. Además, en cada sitio de muestreo se midieron in situ los parámetros $\mathrm{pH}$, temperatura y salinidad, con equipos calibrados YSI pH10 (EcoSense $\left.{ }^{\circledR}\right)$ y ATC/mil-E (Atago $\left.{ }^{\circledR}\right)$.

Las muestras de agua se colectaron según lo indicado en la norma COVENIN:2709 (2002), los sedimentos se tomaron de la capa superficial entre 5 y $10 \mathrm{~cm}$ con una pala de plástico de $1.5 \mathrm{~m}$ de longitud, procurando no alterar los sedimentos que se conservaron en fundas de cierre hermético hasta llegar al laboratorio (Mero et al. 2012).

Para la colecta de gasterópodos se seleccionaron organismos adultos de 3 a $5 \mathrm{~cm}$. En cuanto a las plantas se realizó un análisis preliminar donde se detectó que hojas pequeñas de E. crassipes presentaban baja o nula acumulación de $\mathrm{Cd}$ por lo que se utilizaron hojas de 13 a $16 \mathrm{~cm}$ de largo. Las plantas y los caracoles se colectaron con guantes, se almacenaron en fundas plásticas herméticas y se transportaron al laboratorio.

\section{Proceso de digestión de muestras}

Una vez que las muestras de agua llegaron al laboratorio se acidificaron agregando $1 \mathrm{~mL}$ de $\mathrm{HNO}_{3}$ al $65 \%$ (Merck, Ensure ${ }^{\circledR}$ ) y se filtraron utilizando papel Whatman $\mathrm{N}^{\circ} 40$. Se utilizó el material de referencia QC1132 (agua dulce, Sigma) para la validación de los resultados, con un porcentaje de recuperación de $99.5 \%$.

En el laboratorio los sedimentos fueron extendidos sobre una superficie plástica y secados a temperatura ambiente durante 2 semanas. Posteriormente, fueron disgregados y tamizados a través de un tamiz de $71 \mu \mathrm{m}$. Las muestras tamizadas se secaron en una estufa a $80^{\circ} \mathrm{C}$ por $1 \mathrm{~h}$ para eliminar la humedad y se pesó $1 \mathrm{~g}$ para su posterior digestión por triplicado (Mero et al. 2012).

Para la detección de la fracción total de metales se utilizó el procedimiento propuesto por la Norma EPA3050B (EPA 1996). Se pesó $0.5 \mathrm{~g}$ de muestra, luego se le adicionó $5 \mathrm{~mL}$ de $\mathrm{HNO}_{3}$ al 65 \% (Merck, Ensure $\left.{ }^{\circledR}\right)$ en tubos de ensayos herméticamente sellados, en baño María a $100{ }^{\circ} \mathrm{C}$ por $1 \mathrm{~h}$. Posteriormente, se filtró con papel Whatman $\mathrm{N}^{\circ} 40$ y se enrasó a $100 \mathrm{~mL}$ con agua ultrapura. Paralelamente se usó material de referencia certificado (CRM-016 Trace metals/Freshwater sediment, Sigma) con un porcentaje de recuperación de $101 \%$, como control de calidad. 


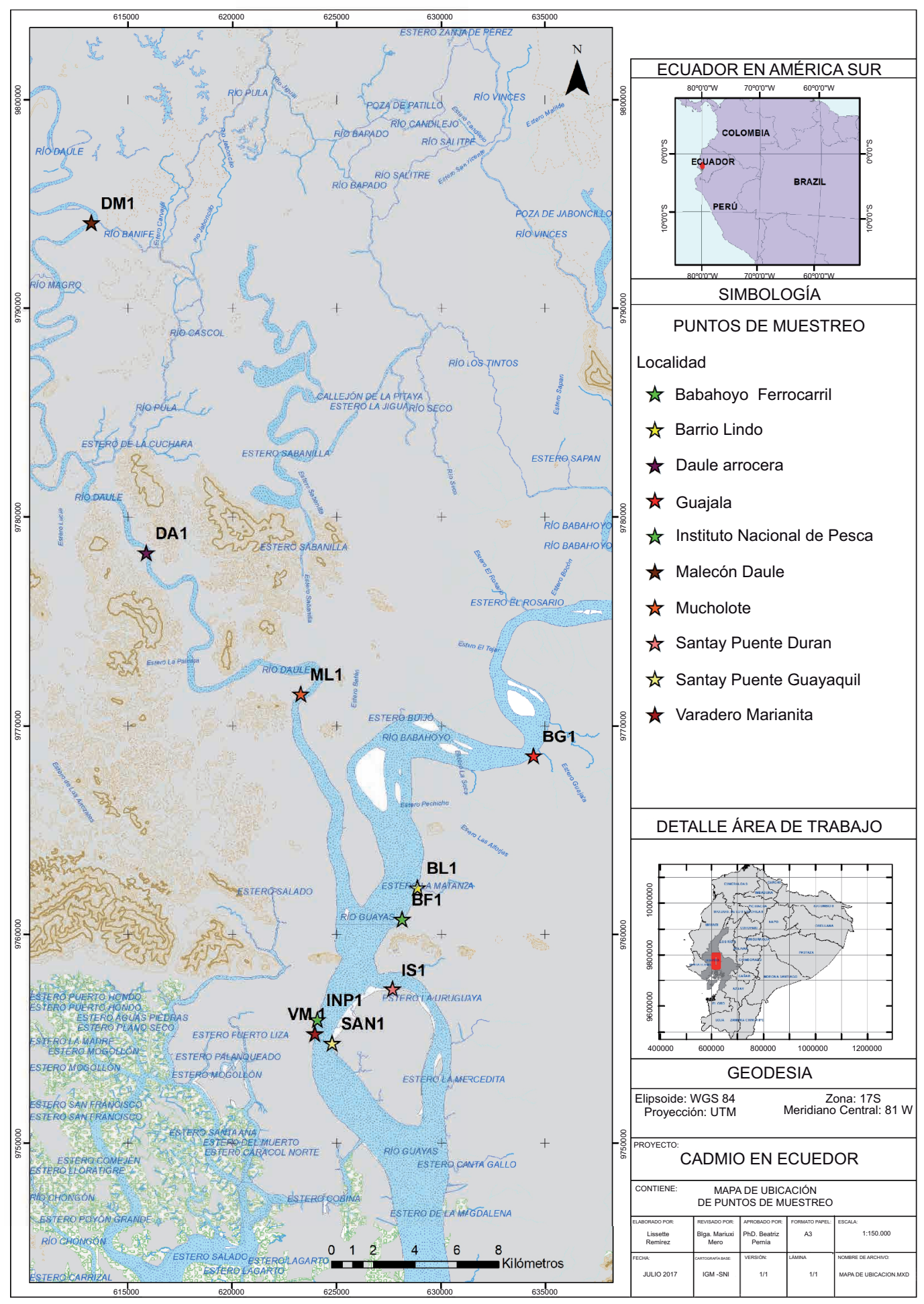

Fig. 1. Área de estudio y estaciones de muestreo en los ríos Guayas, Daule y Babahoyo 
Para la detección de la fracción de Cd biodisponible se utilizó la metodología de Espinosa et al. (2011), quienes indican que a $1 \mathrm{~g}$ de sedimento seco se le añaden $15 \mathrm{~mL}$ de $\mathrm{HCl}$ al $38 \% 0.1 \mathrm{~N}$ (Merck, Grado reactivo ACS) y se deja en reposo durante $24 \mathrm{~h}$, una vez aplicada la metodología se procedió a filtrar con papel Whatman $\mathrm{N}^{\mathrm{o}} 40$ y se enrasó a 50 $\mathrm{mL}$ con el mismo ácido.

Las muestras vegetales se lavaron con agua potable y luego con agua destilada para eliminar todo el material y sedimento adherido. Se realizó la respectiva identificación taxonómica determinando que la especie en estudio era Eichhornia crassipes.

Se separaron las plantas en dos secciones: raíz y hojas, con el fin de determinar la concentración de $\mathrm{Cd}$ en cada parte de la planta. Se eliminó la humedad en estufa a $80^{\circ} \mathrm{C}$, hasta obtener su peso seco, por un periodo de $72 \mathrm{~h}$, luego se procedió a disgregar las muestras y se pesó aproximadamente $1 \mathrm{~g}$ de la muestra por triplicado en vaso de precipitación, a las que se adicionó $4 \mathrm{~mL}$ de ácido nítrico al 65 \% (Merck, Ensure $\left.{ }^{\circledR}\right)$ y se procedió a taparlas con vidrio reloj. Fueron sometidas a calor $\left(90^{\circ} \mathrm{C}\right.$ aproximadamente) en plato calentador hasta eliminación de vapores orgánicos (marrones), luego se añadió $1 \mathrm{~mL}$ de $\mathrm{H}_{2} \mathrm{O}_{2}$ al $30 \%$ (Fisher, Grado reactivo ACS) para acelerar la digestión de la muestra. El extracto resultante se filtró usando papel Whatman No. 40 y se enrasó a un volumen de $50 \mathrm{~mL}$ con agua ultrapura. Como control de calidad se utilizó el material de referencia BCR-670 (Lemna minor, Sigma) con un porcentaje de recuperación de $99 \%$.

Las muestras del gasterópodo fueron lavadas con abundante agua destilada para eliminar restos de sedimentos. Se confirmó que los organismos correspondían a la especie Pomacea canaliculata usando la clave taxonómica de Cuezzo (2009).

Para el procesamiento de digestión se usó la metodología de Alcivar y Mosquera (2011), para lo cual se procedió a retirar el cuerpo blando de los individuos partiendo la concha con un mortero de porcelana. Posteriormente, los cuerpos ya extraídos se colocaron en cápsulas de porcelana y se secaron en una estufa a $80{ }^{\circ} \mathrm{C}$ aproximadamente, durante $48 \mathrm{~h}$. A continuación se trituraron en un mortero y luego fueron llevados a la estufa por una hora para eliminar restos de humedad, pasado este tiempo se procedió a pesar $1 \mathrm{~g}$ de la muestra por triplicado, a la cual se le añadió $3 \mathrm{~mL}$ de $\mathrm{HNO}_{3}$ al $65 \%$ (Merck, Ensure $\left.{ }^{\circledR}\right)$, dejándose digerir en frío por 3 horas, una vez transcurrido este tiempo se realizó la digestión a temperatura de aproximadamente 90 ${ }^{\circ} \mathrm{C}$ en plato calentador hasta eliminación de vapores orgánicos (marrones), luego se filtró utilizando papel Whatman $\mathrm{N}^{\mathrm{o}} 40$ y se enrasaron a $25 \mathrm{~mL}$ con agua ultrapura. Como control de calidad se utilizó el material de referencia BCR-668 (Mussel tissue, Sigma) con un porcentaje de recuperación de $98 \%$.

\section{Técnica de espectrofotometría de absorción atómica}

La cuantificación de $\mathrm{Cd}$ de las muestras de agua, sedimento y organismos, se realizó por la técnica de espectrofotometría de absorción atómica de llama, utilizando un espectrofotómetro modelo AAnalyst100 (Perkin Elmer). Para el control de calidad el equipo fue calibrado usando estándares certificados (Accustandard) y se usó en paralelo material de referencia certificado. Las lecturas de las muestras se realizaron por triplicado para verificar su repetibilidad.

\section{Factor de bioconcentración}

El factor de bioconcentración (FBC) demuestra la capacidad de acumular metales en los organismos. Se determinó utilizando la relación entre el Cd acumulado en la planta o gasterópodo y el Cd presente en el medio según Brooks (1998) y se calculó utilizando la siguiente fórmula:

$\mathrm{FBC}=\frac{\text { Concentracón de } \mathrm{Cd} \text { en la planta o gasterópodo }}{\text { Concentracón de } \mathrm{Cd} \text { en el sedimento }}$

\section{Pruebas estadísticas}

Para comparar las concentraciones promedio de $\mathrm{Cd}$ en agua, sedimentos, Eichhornia crassipes y Pomacea canaliculata se aplicó un análisis de varianza de una vía y una prueba a posteriori de Tukey $(\mathrm{p} \leq 0.05)$. Además, se realizó un análisis de correlación de Pearson para la concentración de $\mathrm{Cd}$ de las especies con el sedimento y un análisis de componentes principales. Todas las pruebas estadísticas se realizaron usando el programa Minitab versión 17.0.

\section{RESULTADOS Y DISCUSIÓN}

\section{Parámetros físicos y químicos en las zonas de estudio}

En el cuadro I se muestran los resultados de temperatura, $\mathrm{pH}$ y salinidad del agua del río Guayas y sus afluentes. En líneas generales la temperatura se encuentra dentro de los límites máximos permisibles para los tres ríos según el Acuerdo Ministerial 097A. La temperatura en las localidades del río Guayas se 
CUADRO I. PARÁMETROS FÍSICOS Y QUÍMICOS: TEMPERATURA $\left({ }^{\circ} \mathrm{C}\right), \mathrm{pH}$ Y SALINIDAD (UPS) DE LAS DIFERENTES LOCALIDADES DE LOS RÍOS GUAYAS: VM (VARADERO LAS MARIANITAS), INP (INSTITUTO NACIONAL DE PESCA), SAN (ISLA SANTAY), RÍO DAULE: ML (MUCHO LOTE), DM (MALECÓN DE DAULE), DA (ARROCERAS DE DAULE) Y RÍO BABAHOYO: BF (LÍNEAS DEL FERROCARRIL), BL (BARRIO LINDO), BG (BARRIO GUAJALA)

\begin{tabular}{llccc}
\hline Río & Localidad & $\mathrm{T}\left({ }^{\circ} \mathrm{C}\right)$ & $\mathrm{pH}$ & Salinidad (ups) \\
\hline Guayas & VM & $27.24 \pm 0.32^{\mathrm{cd}}$ & $6.83 \pm 0.06^{\mathrm{e}}$ & $5.20 \pm 1.30^{\mathrm{a}}$ \\
& $\mathrm{INP}$ & $27.86 \pm 0.53^{\mathrm{bc}}$ & $6.85 \pm 0.02^{\mathrm{e}}$ & $3.80 \pm 0.83^{\mathrm{b}}$ \\
& $\mathrm{SAN}$ & $27.50 \pm 0.21^{\mathrm{cd}}$ & $7.60 \pm 0.17^{\mathrm{abc}}$ & $0.00 \pm 0.00^{\mathrm{c}}$ \\
\hline Daule & $\mathrm{ML}$ & $28.80 \pm 1.07^{\mathrm{ab}}$ & $7.48 \pm 0.45^{\mathrm{cd}}$ & $0.80 \pm 0.83^{\mathrm{c}}$ \\
& $\mathrm{DM}$ & $28.66 \pm 0.33^{\mathrm{ab}}$ & $8.36 \pm 0.10^{\mathrm{a}}$ & $0.00 \pm 0.00^{\mathrm{c}}$ \\
& $\mathrm{DA}$ & $28.06 \pm 0.59^{\mathrm{abc}}$ & $8.15 \pm 0.21^{\mathrm{a}}$ & $0.00 \pm 0.00^{\mathrm{c}}$ \\
\hline Babahoyo & $\mathrm{BF}$ & $29.12 \pm 0.64^{\mathrm{a}}$ & $7.43 \pm 0.40^{\mathrm{d}}$ & $0.00 \pm 0.00^{\mathrm{c}}$ \\
& $\mathrm{BL}$ & $26.38 \pm 0.40^{\mathrm{d}}$ & $8.00 \pm 0.07^{\mathrm{ab}}$ & $0.00 \pm 0.00^{\mathrm{c}}$ \\
& $\mathrm{BG}$ & $27.24 \pm 0.05^{\mathrm{cd}}$ & $7.92 \pm 0.18^{\mathrm{bcd}}$ & $0.00 \pm 0.00^{\mathrm{c}}$ \\
\hline F-Fisher & & 13.42 & 25.64 & 58.25 \\
\hline $\mathrm{p}$ & & 0.000 & 0.000 & 0.000 \\
\hline
\end{tabular}

* Letras iguales indican que no existen diferencias estadísticamente significativas

presentó en el rango de 27.24 a $27.50{ }^{\circ} \mathrm{C}$, en el rio Daule de 28.06 a $28.80^{\circ} \mathrm{C}$ y en el Babahoyo de 26.38 a $29.12^{\circ} \mathrm{C}$.

Por otro lado, el pH en el río Guayas y sus afluentes fluctuó entre 6.83 y 8.35 manteniéndose dentro de los rangos admisibles para la preservación de flora y fauna según el Acuerdo Ministerial 097A. Según Black y Williams (2001) los metales asociados a los sedimentos representan un peligro potencial para el ambiente acuático ya que pueden ser liberados a la columna de agua en caso de alteración de las condiciones físicas y químicas, en especial por acidificación. Según Liu et al. (2013), el Cd presente en los sedimentos podría disolverse y liberar $\mathrm{Cd}$ iónico al agua cuando el $\mathrm{pH}<6$, mientras que cuando es neutro a alcalino, el cadmio adsorbido en el sedimento es estable y se mantiene en esa matriz. De acuerdo con los resultados obtenidos, no se espera que el Cd retenido en los sedimentos sea movilizado a la columna de agua.

En cuanto a la salinidad en la mayoría de las estaciones fueron cero unidades prácticas de salinidad (ups), siendo superior sólo en el río Guayas en las estaciones VM (5.20 \pm 1.30 ups) e INP (3.80 \pm 0.83 ups) presentando diferencias significativas entre ellas $(F=58.25 ; \mathrm{p}=0.000)$. La mayor salinidad del río Guayas se debe a que este río desemboca en el mar y existe un flujo de agua salada dentro del río.

\section{Concentraciones de $\mathrm{Cd}$ en agua y sedimento de los ríos Guayas, Daule y Babahoyo}

Las concentraciones de $\mathrm{Cd}$ en agua no fueron detectables $(<0.028 \mathrm{mg} / \mathrm{L})$ en ninguna de las estaciones de muestreo. Según Zhao et al. (2018) los metales no se mantienen en el agua y son transferidos a los sedimentos por floculación. Dado que el Cd no es un elemento que permanece en la columna de agua, encontrar altos niveles en esa matriz indicaría un aporte activo del mismo por vías antrópicas, lo que no se observó en el presente estudio.

Por el contrario, en los sedimentos del río Guayas y sus afluentes se encontraron elevados niveles de Cd superando los límites máximos permisibles para este metal. En la figura 2 se muestran las concentraciones de $\mathrm{Cd}$ en los sedimentos de las nueve estaciones muestreadas, en las que se observa una alta variabilidad con diferencias estadísticamente significativas $(\mathrm{F}=23.42 ; \mathrm{p}=0.000)$. Además, el 88 $\%$ de los sitios estudiados superaron el límite máximo permitido para $\mathrm{Cd}$ en sedimento según la norma canadiense de $0.67 \mathrm{mg} / \mathrm{kg}$ (CCME 1999) y de 0.50 $\mathrm{mg} / \mathrm{kg}$ en suelo según el Acuerdo Ministerial 097-A de Ecuador (MAE 2015).

En el caso de la estación DA, los valores de cadmio no fueron detectados por el equipo cuyo límite de detección es de $0.028 \mathrm{mg} / \mathrm{kg}$. Estos valores se encuentran por debajo del nivel de fondo para el $\mathrm{Cd}$ en sedimentos de los ríos $(0.1 \mathrm{a} 0.3 \mathrm{mg} / \mathrm{kg})$ según la 


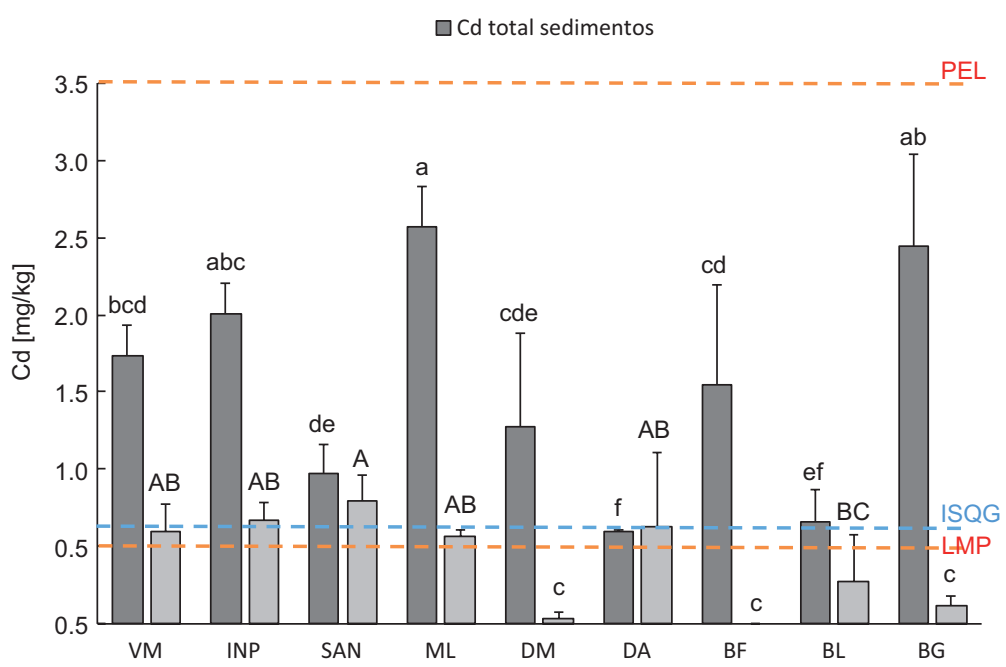

Fig. 2. Concentraciones de $\mathrm{Cd}$ total y biodisponibles en sedimentos en las diferentes localidades de los ríos Guayas: VM (Varadero Las Marianitas), INP (Instituto Nacional de Pesca), SAN (Isla Santay); río Daule: ML (Mucho Lote), DM (Malecón de Daule), DA (Daule Arroceras) y río Babahoyo: BF (Líneas del Ferrocarril), BL (Barrio Lindo), BG (Barrio Guajala). Límite máximo permitido de Cd según la norma ecuatoriana (LMP), nivel de efecto probable (PEL) y valor de referencia canadiense (ISQG). Letras minúsculas iguales indican que no existen diferencias significativas en la concentración total de $\mathrm{Cd}$ entre las localidades y letras mayúsculas iguales indican que no existen diferencias en la concentración de cadmio biodisponible $(\mathrm{p} \geq 0.05)$

tabla de referencia (Screening Quick Reference Table for Inorganics in Fresh water Sediment) emitida por la Administración Oceanográfica y Atmosférica de los Estados Unidos (NOAA, por sus siglas en inglés). Esta estación se encuentra en una zona poco poblada lo que podría explicar las bajas concentraciones de este metal.

Por el contrario, en la estación BG se encontró la mayor contaminación por este elemento, con valores mínimos de $1.79 \mathrm{mg} / \mathrm{kg}$ y máximos de $3.39 \mathrm{mg} / \mathrm{kg}$. En promedio, las concentraciones más altas de $\mathrm{Cd}$ total en sedimentos se presentaron en BG $(2.45 \pm 0.59 \mathrm{mg} / \mathrm{kg})$, $\operatorname{ML}(2.57 \pm 0.26 \mathrm{mg} / \mathrm{kg})$ y en INP $(2.01 \pm 0.19 \mathrm{mg} / \mathrm{kg})$, los cuales superan la normativa ecuatoriana y la canadiense (ISQG, por sus siglas en inglés), la cual indica que por encima de $0.67 \mathrm{mg} / \mathrm{kg}$ de Cd se espera que ocurran efectos biológicos adversos.

Estas zonas se caracterizan por estar muy pobladas y en ellas se encuentran numerosas empresas. La estación ML se localiza cerca del relleno sanitario Las Iguanas y de empresas metalúrgicas, petroleras, fábricas de fertilizantes y plásticos, descritas como fuentes de contaminación por Cd. Además, en el área de estudio se observaron zonas de descargas de aguas residuales sin tratamiento.

Otra posible fuente de contaminación es el uso de fertilizantes fosfatados. El Cd se presenta de manera natural en diversas concentraciones en la roca de fosfato que se extrae para ser utilizada como fertilizante (Mead 2011), razón por la cual se presume que luego de la irrigación y por infiltración llegue a los cuerpos de agua colindantes a las zonas de cultivo. Considerando que de los 8 millones de hectáreas de terrenos agrícolas que se estiman en Ecuador aproximadamente 3 millones de hectáreas corresponden a los suelos que se encuentran en la cuenca del río Guayas, se la considera con alta actividad agrícola, en consecuencia, podría presentarse incidencia de este metal tóxico en el área (SENAGUA 2009).

Según la clasificación del Institute of Soil Science and Plant Cultivation (Wilson y MaliszewskaKordybach 2000) podríamos clasificar la estación DA en nivel 0 o no contaminada, VM, SAN, DM, $\mathrm{BF}, \mathrm{BL}$ en nivel I con concentraciones superiores al límite máximo permitido y las estaciones INP, ML y $\mathrm{BG}$ nivel II ligeramente contaminadas con $\mathrm{Cd}$.

Comparando las concentraciones de $\mathrm{Cd}$ en sedimento de la presente investigación con otros estudios realizados en Ecuador, estas son similares a las halladas en el río Taura (1.42 a $3.75 \mathrm{mg} / \mathrm{kg})$ por Arcos y Castro (2005); inferiores a los reportados en el río Puyango $(44 \mathrm{mg} / \mathrm{kg})$ contaminado por la minería (Tarras-Wahlberg et al. 2001) y superiores 
a los descritos por Carpio (2016) en sedimentos del río Chimbo $(0.09$ a $0.80 \mathrm{mg} / \mathrm{kg})$.

Al contrastar con los resultados de otros ríos a nivel mundial, la concentración de $\mathrm{Cd}$ en el Guayas y sus afluentes son menores a los hallados en ríos como Yenshui en Taiwan, $3.5 \mathrm{mg} / \mathrm{kg}$ (Tsai et al. 2007), Almendares en Cuba, $4.4 \mathrm{mg} / \mathrm{kg}$ (Lima 2005), Gudiamar en España, $16.4 \mathrm{mg} / \mathrm{kg}$ (Kraus y Wiegand 2006), Wyścigi en Polonia, $49.39 \mathrm{mg} / \mathrm{kg}$ (Wojtkowska et al. 2015), Canal Begej en Serbia, $66 \mathrm{mg} / \mathrm{kg}$ (Dalmacija et al. 2006), Seine en Francia, $130 \mathrm{mg} / \mathrm{kg}$ (Meybeck et al. 2007) río Túr en Rumania, $800 \mathrm{mg} / \mathrm{kg}$ (Osán et al. 2007) y Longjiang en China, $5368 \mathrm{mg} / \mathrm{kg}$ (Zhao et al. 2018). Sin embargo, las concentraciones de $\mathrm{Cd}$ son mayores a las reportadas en Sudarban en Bangladesh, $0.82 \mathrm{mg} / \mathrm{kg}$ (Islam et al. 2017), río Casqueiro en Brasil, $1.63 \mathrm{mg} / \mathrm{kg}$ (Harris y Santos 2000) y río Bogotá en Colombia, 0.50 a $1.24 \mathrm{mg} / \mathrm{kg}$ (Lora y Bonilla 2010).

Por otra parte, en la figura 2 se aprecian diferencias en el porcentaje de biodisponibilidad de Cd entre las estaciones de muestreo. La mayor biodisponibilidad de Cd se observó en el sedimento de SAN (81.68 \%) y la menor biodisponibilidad se encontró en $\mathrm{BF}$ (0 \%), DM (2.66 \%), y BG (4.98\%) $(\mathrm{F}=10.42$; $\mathrm{p}=0.000)$. En BG la baja disponibilidad de Cd fue positiva para la biota, ya que de $2.44 \mathrm{mg} / \mathrm{kg}$ total en sedimento sólo se encontró disponible $0.122 \mathrm{mg} / \mathrm{kg}$, valor que se encuentra por debajo de los límites permisibles. Igual resultado se obtuvo en $\mathrm{BF}$, donde existe una alta concentración de $\mathrm{Cd}$ en sedimento que está 2.3 veces por encima de la normativa, pero no se encuentra biodisponible.

Por el contrario, SAN presenta una de las menores concentraciones de Cd total, pero el $81.68 \%$ se encontró biodisponible. Por lo que se recomienda para futuros trabajos analizar no sólo el contenido de Cd total en sedimentos, sino su biodisponibilidad, ya que ésta es la que permite inferir el daño que se puede generar al ecosistema.

Dentro de las posibles consecuencias que podría traer la contaminación por cadmio tenemos la afectación a las especies bentónicas, los peces, las plantas y los humanos. En algunos trabajos se ha demostrado que las especies bentónicas se ven afectadas al exponerse a elevadas concentraciones de Cd (Occhiogrosso et al. 1979, Traunspurger y Drews 1996, Ordonez et al. 2011, Colas et al. 2013). También se ha descrito que este metal genera alteración del ciclo gametogénico de la almeja Ruditapes decussates (Smaoui-Damak et al. 2006) y la reducción de la densidad de macroinvertebrados bentónicos en sedimentos (Ordonez et al. 2011).
En los peces se han reportado numerosos efectos tóxicos del Cd tales como reducción significativa en su actividad natatoria (Eissa et al. 2003), aberraciones cromosómicas, núcleos anormales de células rojas y morfología anormal del esperma (Chandra y KhudaBukhsh 2004).

Es importante destacar que ninguna de las concentraciones de $\mathrm{Cd}$ encontradas en los tres ríos superó el nivel de efecto probable (PEL, por sus siglas en inglés, Probable Effect Level), concentración sobre la cual aparecen con frecuencia efectos biológicos adversos y para el $\mathrm{Cd}$ se ha determinado que en sedimentos la concentración es de $3.5 \mathrm{mg} / \mathrm{kg}$ (LainoGuanes et al. 2015). Sin embargo, deben tomarse medidas para que los niveles de $\mathrm{Cd}$ en los ríos no sigan aumentando y evitar que se generen efectos tóxicos sobre los organismos.

\section{Concentraciones de Cd en Eichhornia crassipes de los ríos Guayas, Daule y Babahoyo}

En E. crassipes se observó acumulación de $\mathrm{Cd}$ tanto en las raíces como en las hojas (Fig. 3 A y B). La concentración de $\mathrm{Cd}$ en las raíces de $\mathrm{BL}, \mathrm{VM}$ y BG fue significativamente mayor que en las de INP, SAN, ML, DM, DA y BF $(F=7.98 ; \mathrm{p}=0.000)$.

Las localidades en donde $E$. crassipes presentó mayor concentración promedio de $\mathrm{Cd}$ en raíces fueron $\mathrm{BL}(8.36 \pm 2.42 \mathrm{mg} / \mathrm{kg}), \mathrm{VM}(5.47 \pm 4.66$ $\mathrm{mg} / \mathrm{kg})$ y BG $(5.76 \pm 1.36 \mathrm{mg} / \mathrm{kg})$ con diferencias significativas con respecto a las localidades de menor concentración DA $(1.31 \pm 0.48 \mathrm{mg} / \mathrm{kg})$ y ML $(0.67$ $\pm 0.46 \mathrm{mg} / \mathrm{kg})(\mathrm{F}=7.98 ; \mathrm{p}=0.000)$. En las hojas no se observaron diferencias significativas en la concentración de $\mathrm{Cd}$ en ocho de nueve localidades, demostrando que las hojas no son el mejor indicador de contaminación por Cd. Sólo se presentó diferencia significativa para la estación BL $(12.72 \pm 7.31 \mathrm{mg} / \mathrm{kg})$ $(\mathrm{F}=10.45 ; \mathrm{p}=0.000)$. En la figura 4 se muestra la distribución espacial de $\mathrm{Cd}$ en E. crassipes en los ríos, donde se aprecian mayores concentraciones en los ríos Babahoyo y Guayas.

Los valores de $\mathrm{Cd}$ encontrados en la presente investigación son mayores a los registrados en raíces de E. crassipes del río Almendares en Cuba donde el mayor valor fue de $3.8 \mathrm{mg} / \mathrm{kg}$ (Lima et al. 2005). La especie E. crassipes se ha propuesto como biomonitora de contaminación por metales y se ha utilizado en monitoreos de ríos en Cuba (Lima et al. 2005) y Venezuela (Schorin et al. 1991), donde se ha comprobado su potencial para detectar metales en los ríos, aun cuando estos se encuentren en bajas concentraciones. En este sentido, Pernía et al. (2016) comprobaron la capacidad de E. crassipes de acumu- 

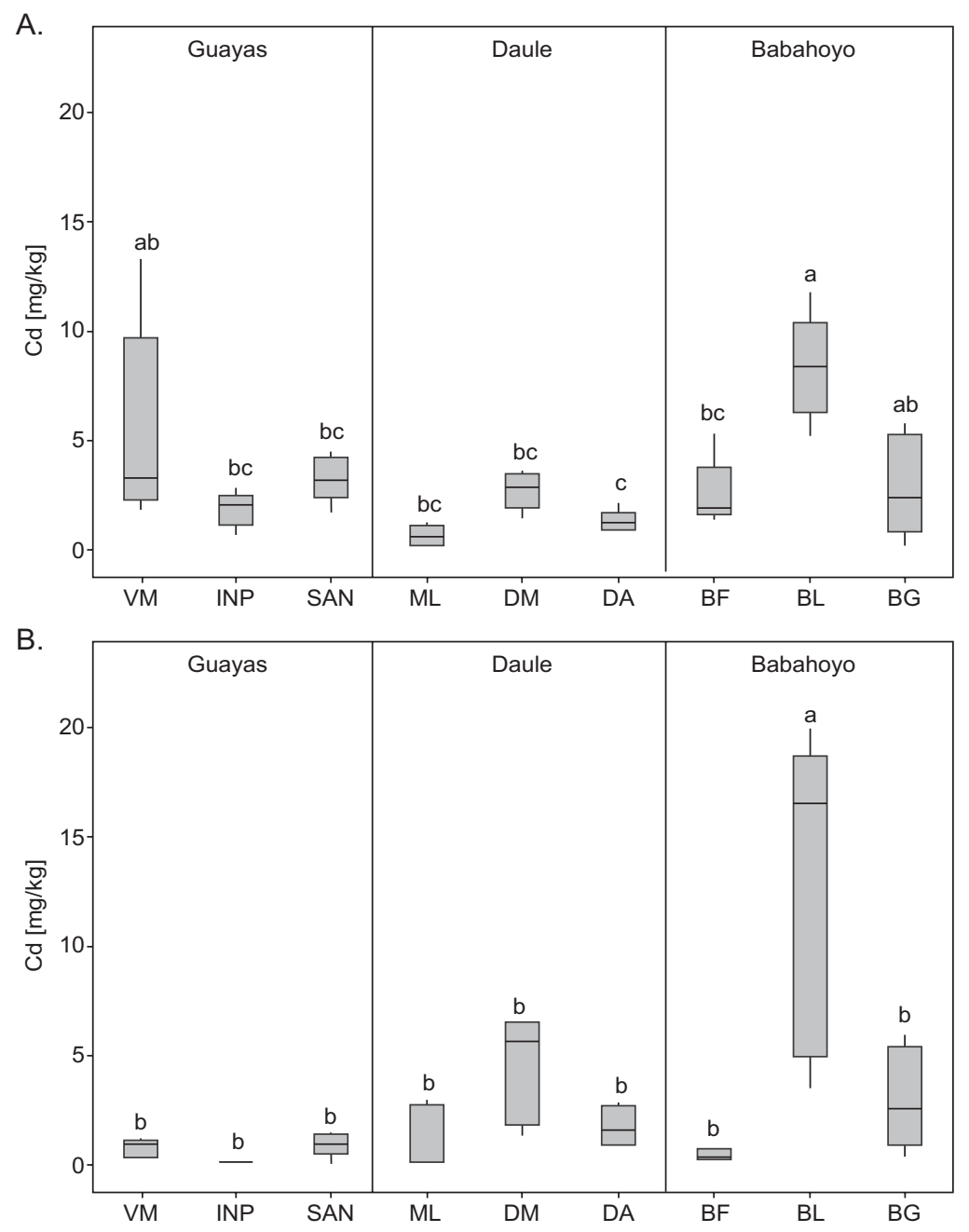

Fig. 3. Concentraciones de Cd en A. raíces y B. hojas de Eichhornia crassipes en las diferentes localidades de los ríos Guayas: VM (Varadero Las Marianitas), INP (Instituto Nacional de Pesca), SAN (Isla Santay); río Daule: ML (Mucho Lote), DM (Malecón de Daule), DA (Arroceras de Daule) y río Babahoyo: BF (Líneas del Ferrocarril), BL (Barrio Lindo), BG (Barrio Guajala). Letras iguales indican que no existen diferencias significativas $(\mathrm{p} \geq 0.05)$.

lar Cd de forma proporcional a la concentración de este metal en el agua, llegando a acumular $514.44 \pm$ $208.11 \mathrm{mg} / \mathrm{kg}$ en hojas y $1482.66 \pm 368.41 \mathrm{mg} / \mathrm{kg}$ en raíces al exponerla a $1 \mathrm{mg} / \mathrm{L}$ de $\mathrm{Cd}$ durante siete días.

Dentro de los efectos negativos que genera el $\mathrm{Cd}$ a la especie E. crassipes se ha descrito reducción de varios parámetros tales como la biomasa, el contenido de pigmentos fotosintéticos, la tasa de asimilación de $\mathrm{CO}_{2}$, la conductancia estomática y la eficiencia en el uso de agua al exponer las plantas a $100 \mathrm{mg} / \mathrm{L}$ de Cd (Zahoor et al. 2018). De igual forma, Pernía et al. (2016) determinaron clorosis en hojas jóvenes de E. crassipes expuestas a $2 \mathrm{mg} / \mathrm{L} \mathrm{de} \mathrm{Cd}$ por siete días.
Sin embargo, a bajas concentraciones del metal (3.5, 7.0, 14.0, y $28.0 \mu \mathrm{M})$, Pereira et al. (2017) demostraron que esta especie es tolerante al $\mathrm{Cd}$, mostrando un incremento en su tasa fotosintética y la conductancia estomática. No obstante, en el presente estudio el Cd no fue detectado por lo que no se puede determinar la tolerancia de la especie a concentraciones inferiores a $0.028 \mathrm{mg} / \mathrm{L}$.

Concentraciones de Cd en Pomacea canaliculata de los ríos Guayas, Daule y Babahoyo

$P$. canaliculata estuvo presente en seis de las nueve localidades. En cuanto al contenido de $\mathrm{Cd}$ en el 


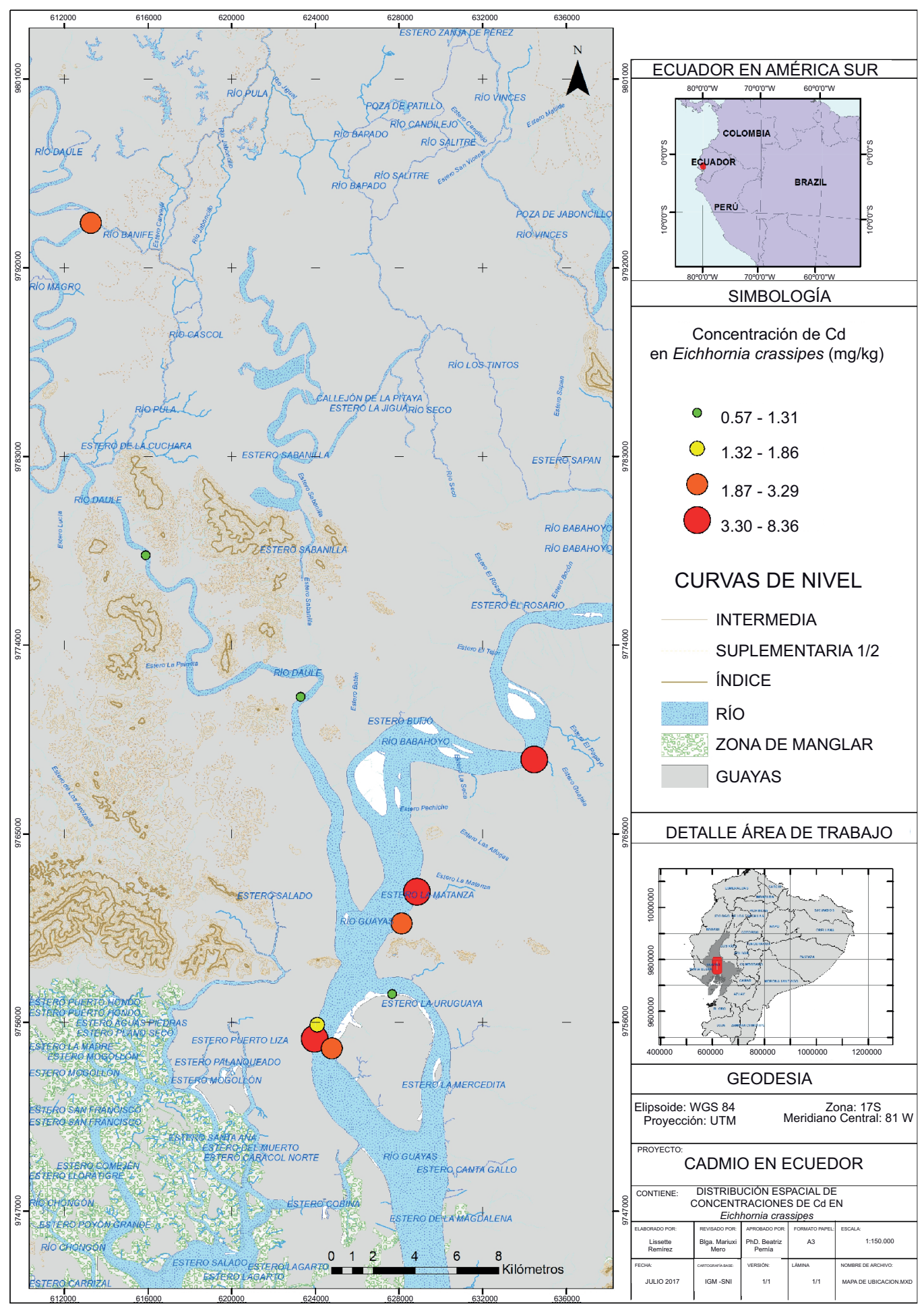

Fig. 4. Distribución espacial de las concentraciones promedio de Cd en Eichhornia crassipes en los ríos Guayas, Daule y Babahoyo. 
tejido de P.canaliculata en la figura 5 se observaron las mayores concentraciones en las estaciones SAN $(1.52 \pm 0.16 \mathrm{mg} / \mathrm{kg}), \mathrm{ML}(1.30 \pm 0.23 \mathrm{mg} / \mathrm{kg})$ y BF $(1.19 \pm 0.16 \mathrm{mg} / \mathrm{kg})$, significativamente mayores a las concentraciones encontradas en las localidades INP, DM y BG $(F=47.52 ; p=0.000)$. En la figura 6 se muestra la distribución espacial de $\mathrm{Cd}$ en el gasterópodo.

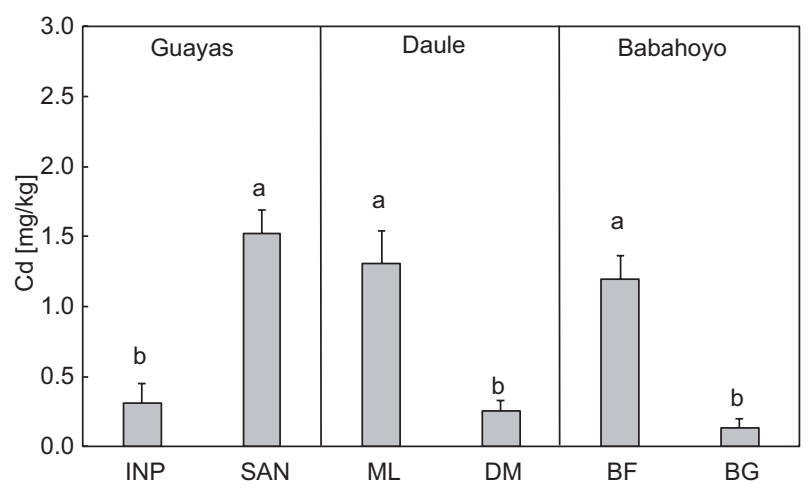

Fig. 5. Concentraciones de Cd en Pomacea canaliculata en las diferentes localidades de los ríos Guayas: VM (Varadero Las Marianitas), INP (Instituto Nacional de Pesca), SAN (Isla Santay); río Daule: ML (Mucho Lote), DM (Malecón de Daule), DA (Arroceras de Daule) y río Babahoyo: BF (Líneas del Ferrocarril), BL (Barrio Lindo), BG (Barrio Guajala). Letras iguales indican que no existen diferencias significativas $(\mathrm{p} \geq 0.05)$.

Estos resultados coinciden con los hallados por Ramírez et al. (2016) quienes encontraron Cd en $P$. canaliculata a razón de $1.221 \pm 0.751 \mathrm{mg} / \mathrm{kg}$ en el río Guayas, $0.954 \pm 0.602 \mathrm{mg} / \mathrm{kg}$ en Daule y 0.738 $\pm 0.598 \mathrm{mg} / \mathrm{kg}$ en Babahoyo. Por el contrario, los resultados del presente estudio son mucho menores a los reportados por Deng et al. (2008) en una mina de China, donde encontraron valores máximos de $30 \mathrm{mg} / \mathrm{kg}$ de $\mathrm{Cd}$ en las vísceras de $P$. canaliculata. Sin embargo, al igual que en este trabajo, Deng et al. (2008) demostraron que las concentraciones acumuladas en $P$. canaliculata no mostraron correlación con el Cd presente en el sedimento por lo que no lo recomiendan como bioindicador de $\mathrm{Cd}$, pero sí como biomonitor.

Comparación de las concentraciones de $\mathrm{Cd}$ encontradas en sedimentos, Eichhornia crasssipes y Pomacea canaliculata

En la figura 7 se observa que $E$. crassipes acumuló mayor concentración de $\mathrm{Cd}$ en comparación con
P. canaliculata en las estaciones INP, SAN, DM y BG $(\mathrm{F}=47.52 ; \mathrm{p}=0.000)$. Por otro lado, la concentración de $\mathrm{Cd}$ en $E$. crassipes fue mayor a la encontrada en el sedimento total en las estaciones SAN, DA, BG y $\mathrm{BL}$, evidenciando que es una excelente acumuladora de Cd. Por el contrario, P. canaliculata sólo acumuló más Cd que E. crassipes en la estación ML y con respecto al sedimento total, las concentraciones fueron menores en INP y ML y en el resto de las estaciones no presentaron diferencias significativas.

\section{Factor de bioconcentración de Cd en Eichhornia crasssipes y Pomacea canaliculata}

Para verificar la capacidad de acumulación de Cd en las especies estudiadas se determinó el factor de bioconcentración (FBC). El FBC de E. crassipes fue mayor a uno en el $88 \%$ de las estaciones indicando su capacidad de acumulación de Cd. El FBC varió significativamente entre las localidades para $E$. crassipes con un valor máximo de $10.86 \pm 4.47$ en $\mathrm{BL}$ y mínimo de $0.26 \pm 0.18$ en $\mathrm{ML}(\mathrm{H}=20.60 ; \mathrm{p}=0.01)$. Por el contrario, el FBC de $P$. canaliculata fue mayor a uno solo en SAN (1.48 \pm 0.13$)$, indicando una menor capacidad de acumulación de Cd en comparación con E. crassipes (Cuadro II).

Correlación entre los parámetros físicos y químicos y el contenido de cadmio en el sedimento, Eichhornia crassipes y Pomacea canaliculata

Para evaluar el grado de asociación de los parámetros físicos y químicos con la concentración de $\mathrm{Cd}$ en sedimento y organismos se aplicó un análisis de correlación de Pearson donde se determinó una fuerte correlación entre el Cd presente en E. crassipes y la temperatura $(\mathrm{r}=0.627 ; \mathrm{p}=0.000)$, lo que indica que, a mayor temperatura mayor concentración de cadmio acumulada en las plantas. Según Zhuo et al. (2013) temperaturas de $30{ }^{\circ} \mathrm{C}$ pueden incrementar la biodisponibilidad del Cd en los sedimentos y ser absorbido por las plantas. En este sentido, Hooda y Halloway (1993) demostraron que la biodisponibilidad del $\mathrm{Cd}$ en los suelos es proporcional a la temperatura, ya que al incrementar la temperatura aumenta la solubilidad de los compuestos.

Asimismo, se observó una relación débil inversamente proporcional entre el $\mathrm{pH}$ y el $\mathrm{Cd}$ en sedimento $(\mathrm{r}=-0.411 ; \mathrm{p}=0.005)$ y el Cd biodisponible $(\mathrm{r}=-0.411 ; \mathrm{p}=0.005)$, mostrando que a mayor $\mathrm{pH}$ el Cd se encuentra menos biodisponible. $\mathrm{Se}$ ha descrito que el $\mathrm{pH}$ afecta el equilibrio entre la especiación metálica, solubilidad, adsorción e intercambio de iones en el suelo y se ha considerado 


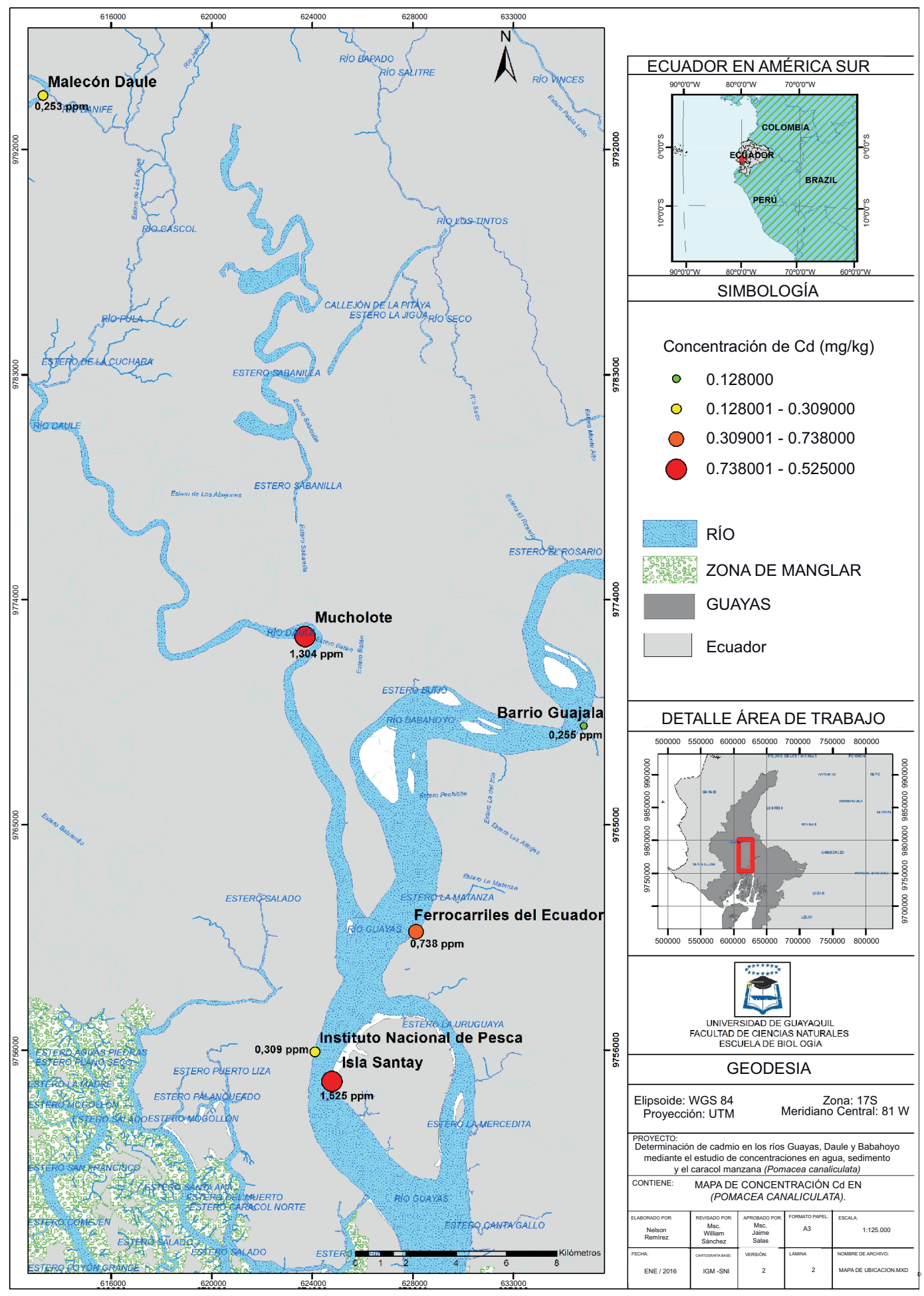

Fig. 6. Distribución espacial del Cd en Pomacea canaliculata en los ríos Guayas, Daule y Babahoyo 


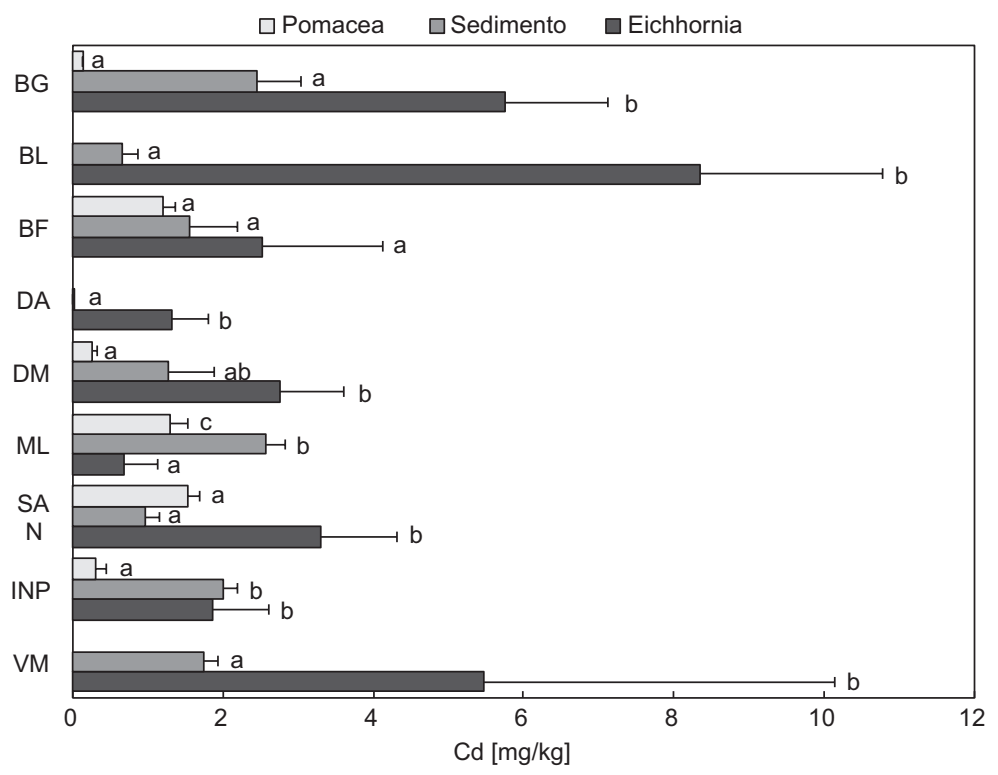

Fig. 7. Comparación de la concentración de Cd hallada en sedimento, raíces de Eichhornia crassipes y Pomacea canaliculata por localidades de los ríos Guayas, Daule y Babahoyo. Los resultados se muestran como media \pm desviación estándar $(n=5)$. Letras iguales indican que no existen diferencias estadísticamente significativas entre las concentraciones de $\mathrm{Cd}$ en los sustratos por localidad $(\mathrm{p} \geq 0.05)$

CUADRO II. FACTOR DE BIOCONCENTRACIÓN DE CADMIO DE Eichhornia crassipes Y Pomacea canaliculata POR ESTACIONES DE MUESTREO EN LAS LOCALIDADES DE LOS RÍOS GUAYAS: VM (VARADERO LAS MARIANITAS), INP (INSTITUTO NACIONAL DE PESCA), SAN (ISLA SANTAY); RÍO DAULE: ML (MUCHO LOTE), DM (MALECÓN DE DAULE), DA(ARROCERAS DE DAULE) Y RÍO BABAHOYO: BF (LÍNEAS DEL FERROCARRIL), BL (BARRIO LINDO), BG (BARRIO GUAJALA). $(\mathrm{N}=5)$. LOS RESULTADOS SE MUESTRAN COMO MEDIA \pm DESVIACIÓN ESTÁNDAR $(\mathrm{N}=5)$

\begin{tabular}{llcccc}
\hline Río & Localidad & FBC Eichhornia & FBC Pomacea & F-Fisher & $\mathrm{p}$ \\
\hline \multirow{2}{*}{ Guayas } & VM & $2.02 \pm 1.17$ & NR & - & - \\
& INP & $0.95 \pm 0.41^{\mathrm{a}}$ & $0.16 \pm 0.06^{\mathrm{b}}$ & 10.40 & 0.018 \\
& $\mathrm{SAN}$ & $3.34 \pm 0.68^{\mathrm{a}}$ & $1.48 \pm 0.13^{\mathrm{b}}$ & 20.71 & 0.004 \\
\hline \multirow{2}{*}{ Daule } & $\mathrm{ML}$ & $0.26 \pm 0.18^{\mathrm{a}}$ & $0.53 \pm 0.06^{\mathrm{a}}$ & 3.75 & 0.111 \\
& $\mathrm{DM}$ & $1.89 \pm 1.07^{\mathrm{a}}$ & $0.44 \pm 0.50^{\mathrm{a}}$ & 4.57 & 0.085 \\
\hline Babahoyo & $\mathrm{BF}$ & $1.70 \pm 1.16^{\mathrm{a}}$ & $0.80 \pm 0.33^{\mathrm{a}}$ & 1.60 & 0.274 \\
& $\mathrm{BL}$ & $10.86 \pm 4.47$ & $\mathrm{NR}$ & - & - \\
& $\mathrm{BG}$ & $2.50 \pm 1.03^{\mathrm{a}}$ & $0.05 \pm 0.00^{\mathrm{a}}$ & 4.71 & 0.076 \\
\hline
\end{tabular}

* Letras iguales indican que no existen diferencias estadísticamente significativas entre el FBC de Eichhornia crassipes y el FBC de Pomacea canaliculata $(\mathrm{p} \geq 0.005), \mathrm{NR}=$ no registrado

el factor más importante en la biodisponibilidad de los metales (McBride et al. 1997). A pH básicos los metales son removidos de la solución del suelo y adsorbidos por los coloides, disminuyendo su biodisponibilidad (Alloway 1995). En este sentido, los ríos presentan un $\mathrm{pH}$ de neutro a básico lo que reduce su disponibilidad para la biota y su potencial toxicidad.

También se apreció una correlación débil entre $\mathrm{Cd}$ biodisponible en el sedimento y la salinidad del agua ( $\mathrm{r}=0.334 ; \mathrm{p}=0.025)$. Se ha demostrado que a mayor salinidad, mayor biodisponibilidad de $\mathrm{Cd}$, ya 
que los cloruros generan compuestos solubles con el metal (García y Dorronsoro 2005).

De igual forma, se realizó un análisis de componentes principales donde se evidenció que cuatro de ellos explicaron el $90 \%$ de la correlación entre variables. El primer componente, CP1, muestra la correlación entre la concentración de $\mathrm{Cd}$ en $E$. crassipes y el $\mathrm{pH}$ (Fig. 8). Por otro lado, el CP2 evidenció relación entre la concentración de $\mathrm{Cd}$ en el gasterópodo y la temperatura. E1 CP3 mostró correlación entre la biodisponibilidad del $\mathrm{Cd}$ en sedimento y la temperatura y finalmente en CP4 se observó la relación del Cd en el sedimento con la salinidad.

Estos resultados explican la variación en la acumulación de $\mathrm{Cd}$ en E. crassipes en las estaciones como consecuencia de las variaciones en los parámetros físicos y químicos y cómo estos afectan la biodisponibilidad del cadmio en los sedimentos de los ríos.

Dentro de las limitantes de esta investigación tenemos que se utilizó un espectrofotómetro de absorción atómica cuyo límite de detección de cadmio es $0.028 \mathrm{mg} / \mathrm{L}$ por lo que no se pudo apreciar el contenido de este metal en agua. Se recomienda para futuros estudios utilizar un equipo más sensible (espectrofotometría acoplada a horno de grafito o espectroscopía de emisión por plasma de acoplamiento inductivo). Otra recomendación es determinar la concentración de materia orgánica en el sedimento ya que podría explicar la biodisponibilidad del metal en este sustrato, así como también realizar la granulometría del sedimento ya que los metales suelen acumularse en la fracción fina.

Finalmente, se propone el uso de E. crassipes como especie biomonitora de contaminación por Cd en los ríos Guayas, Daule y Babahoyo por presentar las ventajas de estar distribuida en todas las estaciones estudiadas, en cantidad abundante durante todo el año, además de acumular altas concentraciones del metal y presentar un alto factor de bioconcentración.

\section{CONCLUSIONES}

No se detectó Cd en el agua de ninguna de las estaciones, por el contrario, ocho de las nueve

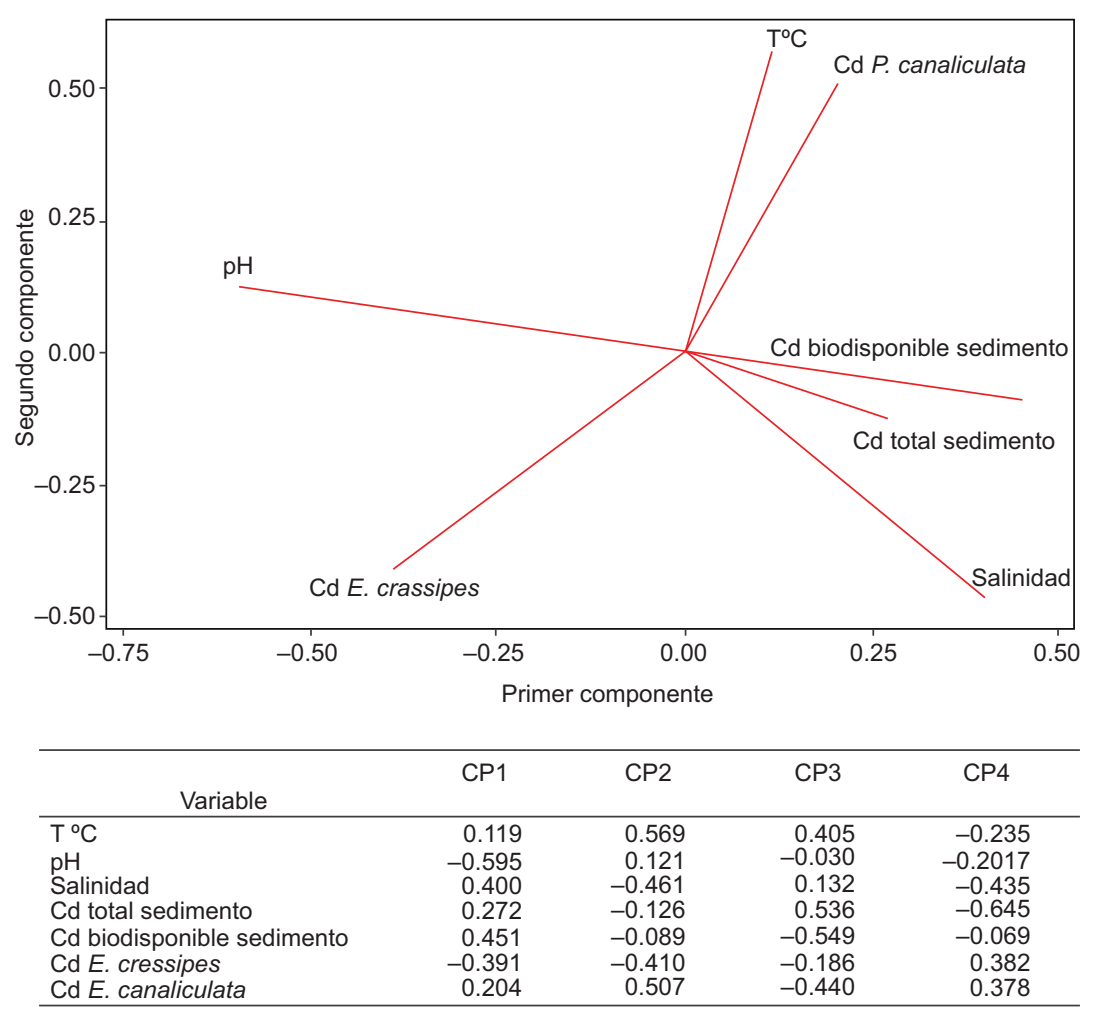

Fig. 8. Análisis de componentes principales entre todos los parámetros analizados: temperatura $\left(\mathrm{T}^{\circ} \mathrm{C}\right), \mathrm{pH}$, salinidad, $\mathrm{Cd}$ total en sedimento, $\mathrm{Cd}$ biodisponible en sedimento, $\mathrm{Cd}$ en $E$. crassipes y $\mathrm{Cd}$ en $P$. canaliculata. $\mathrm{CP}=$ componente principal 
localidades estudiadas en los ríos Guayas, Daule y Babahoyo superan los límites máximos permisibles de $\mathrm{Cd}$ en sedimentos según las normas canadiense y ecuatoriana; sin embargo, el metal presentó una baja biodisponibilidad en dicho sedimento, con valores inferiores al ISQG y PEL, minimizando los posibles daños a la biota. Aun así, los organismos fueron capaces de acumular el Cd en sus tejidos y la especie E. crassipes demostró ser una buena biomonitora, a diferencia de $P$. canaliculata quien presentó un menor factor de bioacumulación.

También se observó correlación entre los parámetros temperatura y $\mathrm{pH}$ con la concentración de $\mathrm{Cd}$ en los organismos y entre la salinidad con la biodisponibilidad del metal en el sedimento.

Por otro lado, se apreciaron diferencias significativas en los niveles de $\mathrm{Cd}$ entre localidades de un mismo río indicando fuentes puntuales de contaminación por lo que se recomienda a las autoridades del ambiente realizar monitoreos anuales para controlar la contaminación por metales en estos ríos, analizando sedimentos (totales y biodisponibles) y la especie E. crassipes.

\section{AGRADECIMIENTOS}

Los autores agradecen al Programa Prometeo de la Secretaría de Educación Superior, Ciencia, Tecnología e Innovación (SENESCYT) por el financiamiento de parte de los reactivos y al Ministerio del Ambiente de Ecuador (MAE) por la autorización de investigación Científica $\mathrm{N}^{\circ}$ 015-IC-FLO/FAUDPG/MAE.

\section{REFERENCIAS}

Alcivar M. y Mosquera J. (2011). Concentración de metales pesados ( $\mathrm{Cr}$ total, $\mathrm{Pb}, \mathrm{Cd}$ ) en agua superficial y sedimentos en el Estero Salado (Guayaquil). Tesis de Licenciatura. Facultad de Ciencias Naturales, Universidad de Guayaquil. Guayaquuil, Ecuador, 56 pp.

Alloway B.J. (1995). Soil processes and behaviour of metals. En: heavy metals in soils (B.J. Alloway, Ed). John Wiley, Nueva York, EUA, pp.11-37.

Arcos V. y Castro R. (2005). Metales pesados en agua, sedimentos y organismos. Revista Científica de Ciencias Naturales y Ambientales 1 (1), 103-120.

Black M.C. y Williams P.L. (2001). Preliminary assessment of metal toxicity in the middle tisza river (Hungary) flood plain. J. Soils Sediments 1 (4), 213-216. DOI: 10.1007/BF02987727
Brooks R. R. (1998). Plants that hyperaccumulate heavy metals. Their role in phytoremediation, microbiology archaeology, mineral exploration and phytomining. CABI. Wallingford, Reino Unido, 380 pp.

Burger J. (2008). Assessment and management of risk to wildlife from cadmium. Sci. Total Environ. 389 (1), 37-45. DOI: 10.1016/j.scitotenv.2007.08.037

Carpio Y. (2016). Cuantificación de cadmio (Cd) y plomo $\mathrm{Pb}$ en agua, sedimento y plantas en el río Chimbo del cantón Marcelino Maridueña, Prov. Guayas. Tesis de Maestría. Facultad de Ciencias Naturales, Universidad de Guayaquil. Guayaquil, Ecuador, 112 pp.

Castrillo M., Pernía B., De Sousa A. y Reyes R. (2012). Utilization of different aspects associated with cadmium tolerance in plants to compare sensitive and bioindicator species. En: Phytotechnologies: remediation of environmental contaminants (N. Anjum, M.E. Pereira, I. Ahmad, A.C. Duarte, S. Umar y N.A. Khan, Eds.). CRC Press, Nueva York, EUA, pp. 427-440.

CCME (1999). Guía canadiense de calidad del suelo para la protección del medio ambiente y la salud humana. The Canadian Council of Ministers of the Environment [en línea]. http://ceqg-rcqe.ccme.ca/download/ en/261 07/01/2017

Chandra P. y Khuda-Bukhsh A.R. (2004). Genotoxic effects of cadmium chloride and azadirachtin treated singly and in combination in fish. Ecotox. Environ. Safe. 58 (2), 194-201.

DOI: 10.1016/j.ecoenv.2004.01.010

Clemens S., Aarts M. G. M., Thomine S. y Verbruggen N. (2013). Plant science: The key to preventing slow cadmium poisoning. Trends Plant Sci. 18 (2), 92-99. DOI: $10.1016 /$ j.tplants.2012.08.003

Colas F., Archaimbault V., Férard J.F., Bouquerel J., Roger M.C. y Devin S. (2013). Benthic indicators of sediment quality associated with run-of-river reservoirs. Hydrobiologia 703 (1), 149-164.

DOI: $10.1007 / \mathrm{s} 10750-012-1355-\mathrm{y}$

COVENIN (2002). COVENIN 2709-2002. Aguas naturales, industriales y residuales. Guía para las técnicas de muestreo. 1era revisión. Editorial Fondonorma. Caracas, Venezuela. 28 de mayo de 2002.

Cuezzo M. G. (2009). Mollusca: Gastropoda. En: Macroinvertebrados bentónicos sudamericanos: sistemática y biología. (E. Domínguez y H. Fernández, Eds.). Fundación Miguel Lillo, Tucumán, Argentina, pp. 595-629.

Dalmacija B., Prica M., Ivancev-Tumbas I., van der Kooij A., Roncevic S., Krcmar D. y Teodorovic I. (2006). Pollution of the Begej canal sediment-metals, radioactivity and toxicity assessment. Environment International 32 (5), 606-615.

DOI: 10.1016/J.ENVINT.2006.01.006 
Deng P.Y., Shu W.S., Lan Y. y Liu W. (2008). Metal contamination in the sediment, pondweed, and snails of a stream receiving effluent from a lead/zinc mine in southern China. Bull. Environ. Contam. Toxicol. 81 (1), 69-74. DOI: 10.1007/s00128-008-9428-3

Eissa B.L., Salibiani A., Ferrari L., Porta P. y Borgnia M. (2003). Evaluación toxicologica no invasiva del cadmio: modificaciones de biomarcadores conductuales en Cyprinus carpio. Biología Acuática 20, 56-62.

EPA (1996). Method 3050B: acid digestion of sediments, sludges, and soils. Environmental Protection Agency. Revision 2. Washington, EUA. Diciembre de 1996.

Espinosa L. F., Parra J. P. y Villamil C. (2011). Determinación del contenido de metales pesados en las fracciones geoquímicas del sedimento superficial asociado a los manglares de la ciénaga grande de Santa Marta, Colombia. Boletín de Investigaciones Marinas y Costeras 40 (1), 7-23.

Fernández-Cadena J. C., Andrade S., Silva-Coello C. L. y De la Iglesia R. (2014). Heavy metal concentration in mangrove surface sediments from the north-west coast of South America. Mar. Pollut. Bull. 82 (1 - 2), 221-226. DOI: 10.1016/j.marpolbul.2014.03.016

Filipič M. (2012). Mechanisms of cadmium induced genomic instability. Mutat. Res. 733 (1 -2), 69-77. DOI: $10.1016 /$ j.mrfmmm.2011.09.002

Fuentes M., Astudillo L., Diaz A. y Martínez G. (2010). Distribución de metales pesados en los sedimentos superficiales del saco del Golfo de Cariaco, Sucre, Venezuela. Rev. Biol. Trop. 58 (3), 129-140.

García I. y Dorronsoro C. (2005). Contaminación por metales pesados. En: Tecnología de suelos. Universidad de Granada. Departamento de Edafología y Química Agrícola. Granada, España [en línea]. http://edafologia. ugr.es/conta/tema15/introd.htm 10/05/2017.

Harris R. R. y Santos M. C. F. (2000). Heavy metal contamination and physiological variability in the Brazilian mangrove crabs Ucides cordatus and Callinectes danae (Crustacea: Decapoda). Mar. Biol.137 (4), 691-703.

DOI: $10.1007 / \mathrm{s} 002270000382$

Henson M.C. y Chedrese P. (2004). Endocrine disruption by cadmium, a common environmental toxicant with paradoxical effects on reproduction. Exp. Biol. Med. 229 (5), 383-392.

Huayamabe J. (2013). Estudio de las aguas y sedimento del río Daule, en la provincia del Guayas, desde el punto de vista físico químico, orgánico, bacteriológico y toxicológico.Tesis Doctoral. Universidad de las Palmas de Gran Canaria. Las Palmas, España, 322 pp.

Hooda P.S. y Alloway B.J. (1993). Effect of time and temperatura on the bioavailability of $\mathrm{Cd}$ and $\mathrm{Pb}$ from sludge-amended soil. J. Soil. Sci. 44, 97-110.
Islam M. A., Al-mamun A., Hossain F., Quraishi S. B., Naher K., Khan R. y Tamim U. (2017). Contamination and ecological risk assessment of trace elements in sediments of the rivers of Sundarban mangrove forest, Bangladesh. Mar. Pollut. Bull. 124 (1), 356-366. DOI: 10.1016/j.marpolbul.2017.07.059

Kaushik A., Kansal A., Santosh M., Kumari S. y Kaushik C. P. (2009). Heavy metal contamination of river Yamuna, Haryana, India: assessment by metal enrichment factor of the sediments. J. Hazard. Mater. 164 (1), 265270. DOI: 10.1016/j.jhazmat.2008.08.031

Kraus U. y Wiegand J. (2006). Long-term effects of the Aznalcóllar mine spill-heavy metal content and mobility in soils and sediments of the Guadiamar river valley (SW Spain). Sci. Total Environ. 367 (2 - 3), 855871. DOI: 10.1016/j.scitotenv.2005.12.027

Laino-Guanes R., Bello-Mendoza R., González-Espinosa M., Jiménez-Otárola F. y Musalém-Castillejos K. (2015). Concentración de metales en agua y sedimentos de la cuenca alta del río Grijalva, frontera MéxicoGuatemala. Tecnología y Ciencias del Agua 6 (4), 61-74.

Lima L., Olivares-Rieumont S., Columbie I., de la Rosa Mederos, D. y Gil R. (2005). Niveles de plomo, zinc, cadmio y cobre en el rio Almendares, Ciudad Habana, Cuba. Rev. Int. Contam. Ambie. 21 (3), 115-124.

Liu D., Zhang C., Chen X., Yang Y., Wang S., Li Y., Hu H. Ge Y. y Cheng W. (2013). Effects of pH, Fe, and $\mathrm{Cd}$ on the uptake of $\mathrm{Fe}^{2+}$ and $\mathrm{Cd}^{2+}$ by rice. Environ. Sci. Pollut. R. 20 (12), 8947-8954. DOI: $10.1007 / \mathrm{s} 11356-013-1855-\mathrm{y}$

Lora R. y Bonilla H. (2010). Remediación de un suelo de la cuenca alta del río Bogotá contaminado con los metales pesados cadmio y cromo. Revista U.D.C.AActualidad y Divulgación Científica 13 (2), 61-70.

MAE (2015). Acuerdo Ministerial 097A, Texto Unificado de Legislación Secundaria de Ecuador. Ministerio del Ambiente de Ecuador. Quito, Ecuador. 4 de noviembre de 2015.

McBride M., Sauve S. y Hendershot W. (1997). Solubility control of $\mathrm{Cu}, \mathrm{Zn}, \mathrm{Cd}$ and $\mathrm{Pb}$ in contaminated soils. Eur. J. Soil Sci. 48 (2), 337-346.

DOI: $10.1111 / j .1365-2389.1997 . t b 00554 . x$

Mead N. (2011). Confusión por el cadmio: ¿Los consumidores necesitan protección? Salud Pública México 53 (2), 178-186. DOI: $10.1590 / \mathrm{S} 0036-36342011000200011$

Mero M., Arcos V., Egas F., Siavichay R. y Lindao G. (2012). Determinación de metales pesados $(\mathrm{Cd} \mathrm{y} \mathrm{Pb})$ en moluscos bivalvos de interés comercial Anadara tuberculosa y A. grandis de Puerto El Morro, Ecuador. Revista de Divulgación de la Dirección de Investigaciones y Proyectos Académicos de La Universidad de Guayaquil 4 (4), 19-32. 
Meybeck M., Lestel L., Bonté P., Moilleron R., Colin J. L., Rousselot O. y Thévenot D. R. (2007). Historical perspective of heavy metals contamination $(\mathrm{Cd}, \mathrm{Cr}, \mathrm{Cu}$, $\mathrm{Hg}, \mathrm{Pb}, \mathrm{Zn}$ ) in the Seine river basin (France) following a DPSIR approach (1950 - 2005). Sci. Total Environ. $375(1$ - 3), 204-231.

DOI: $10.1016 /$ j.scitotenv.2006.12.017

Muñoz J. (2017). Determinación de cadmio en fertilizantes, plantas de Oryza sativa L. y suelos de la provincia del Guayas: propuesta de saneamiento. Tesis de Ingeniería. Facultad de Ciencias Naturales, Universidad de Guayaquil. Guayaquil, Ecuador, 58 pp.

Occhiogrosso T.J., William W. y Gerald L. (1979). Effects of heavy metals on benthic macroinvertebrate densities in foundry cove on the Hudson river. B. Environ. Contam. Tox. 22 (1), 230-237.

Ordonez C., Lougheed V. L., Gardea-torresdey J. L. y Bain L. J. (2011). Impact of metals on macroinvertebrate assemblages in the forgotten stretch of the río Grande. Arch. Environ. Con. Tox. 60 (3), 426-436 . DOI: $10.1007 / \mathrm{s} 00244-010-9557-7$

Osán J., Török S., Alföldy B., Alsecz A., Falkenberg G., Baik S. Y. y Van Grieken R. (2007). Comparison of sediment pollution in the rivers of the Hungarian upper Tisza region using non-destructive analytical techniques. Spectrochim Acta B: Atomic Spectroscopy 62 (2), 123-136.

DOI: $10.1016 /$ J.SAB.2007.02.005

Paul D. (2017). Research on heavy metal pollution of river Ganga: a review. Annals of Agrarian Science 15 (2), 278-286. DOI: 10.1016/j.aasci.2017.04.001

Perdomo L., Ensminger I., Espinosa L. fernanda, Elster C., Wallner-kersanach M. y Schnetter M.L. (1999). The mangrove ecosystem of the ciénaga Grande de Santa Marta (Colombia): observations on regeneration and trace metals in sediment. Mar. Pollut. Bull. 37 (8 - 12), 393-403. DOI: 10.1016/S0025-326X(99)00075-2

Pereira F.J., De Castro E.M., Pires M.F., De Oliveira C. y Pasqual M. (2017). Anatomical and physiological modifications in water hyacinth under cadmium contamination. J. Appl. Bot. Food Qual. 90, 10-17. DOI: 10.5073/JABFQ.2017.090.003

Pernía B., Mero M., Muñoz J., Bravo K., Morán N., Zambrano J. yTorres G. (2016). Plantas acuáticas con potencial para fitoextracción de cadmio en arrozales del cantón Daule, provincia del Guayas, Ecuador. Revista Científica de Ciencias Naturales y Ambientales 10 (2), 25-30.

Pernía B., Sousa A. D. E., Reyes R. y Castrillo M. (2008). Biomarcadores de contaminación por cadmio en plantas. Interciencia 33 (2), 112-119.

Proaño M. (2016). Análisis espacial de concentraciones de metales pesados en agua y sedimentos en la Reserva
Ecológica Manglares de Churute. Tesis de Maestría. Facultad de Ciencias Naturales, Universidad de Guayaquil. Guayaquil, Ecuador, 60 pp.

Ramírez N., Pernía B., Mero M., Larreta E., NoboaCárdenas A., Ramírez-Moreira L. y Castro R. (2016). Pomacea canaliculata (Lamarck, 1828) como bioindicador de la presencia de cadmio en el río Guayas, Daule y Babahoyo. Revista Científica de Ciencias Naturales y Ambientales 10 (1), 19-28.

Schorin H., Benzo Z. A., Bastidas C. y Quimica C. D. (1991). The use of water hyacinths to determine trace metal concentrations in the tropical Morichal Largo River, Venezuela. Appl. Geochem. 6 (2), 195-200. DOI: 10.1016/0883-2927(91)90029-O

SENAGUA (2009). Problemática y conflictos sobre los recursos hídricos por efectos del cambio climático [en línea]. https://www.utpl.edu.ec/obsa/wp-content/uploads/2012/09/1_ded-senagua_portadas.pdf 06/06/2017.

Smaoui-Damak W., Rebai, T., Berthet B. y Hamza-Chaffai A. (2006). Does cadmium pollution affect reproduction in the clam Ruditapes decussatus? A one-year case study. Comp. Biochem. Phys. C: Toxicology and Pharmacology 143 (2), 252-261.

DOI: $10.1016 /$ j.cbpc.2006.02.009

Tarras-Wahlberg N. H., Flachier A., Lane S. N. y Sangfors O. (2001). Environmental impacts and metal exposure of aquatic ecosystems in rivers contaminated by small scale gold mining: The Puyango River basin, southern Ecuador. Sci. Total Environ. 278 (1 - 3), 239-261. DOI: $10.1016 / \mathrm{S} 0048-9697(01) 00655-6$

Traunspurger W. y Drews C. (1996). Toxicity analysis of freshwater and marine sediments with meio and macrobenthic organisms: a review. Hydrobiologia 328 (3), 215-261.

Tsai L., Yu K. y Ho S.T. (2007). Cadmium distribution in sediment profiles of the six main rivers in southern Taiwan. J. Hazard. Mater.148 (3), 630-639.

DOI: 10.1016/J.JHAZMAT.2007.03.024

Wilson M. J. y Maliszewska-Kordybach B. (2000). Soil quality, sustainable agriculture and environmental security in central and eastern europe. Springer Netherlands, Dordrecht, Países Bajos, 375 pp.

Wojtkowska M., Karwowska E., Chmielewska I., Bekenova K. y Wanot E. (2015). Copper and cadmium in bottom sediments dredged from Wyścigi Pond, Warsaw, Poland - contamination and bioaccumulation study. Environ. Monit. Assess. 187 (12), 737. DOI: $10.1007 / \mathrm{s} 10661-015-4945-0$

Zahoor A., Ahmad F., Hameed M. y Ahmad S. (2018). Structural and functional aspects of photosynthetic response in Eichhornia crassipes (Mart.) Solms under cadmium stress. Pak. J. Bot. 50 (2), 489-493. 
Zhao X., Yao L., Ma Q., Zhou G., Wang L. y Fang Q. (2018). Distribution and ecological risk assessment of cadmium in water and sediment in Longjiang River, China: Implication on water quality management after pollution accident. Chemosphere 194, 107-116.

DOI: 10.1016/j.chemosphere.2017.11.127
Zhuo Q.F., Xu Z.C., Guo Q.W., Yi H., Wang Z.X. y Liu W.R. (2013). Estimation of the stability of cadmium flocs. Chinese Environmental Science 33, 245-324. 